\title{
Anticoagulation Control of Warfarin in Pharmacist-Led Clinics Versus Physician-Led Clinics: A Prospective Observational Study
}

This article was published in the following Dove Press journal: Risk Management and Healthcare Policy

\author{
Sultan Alghadeeer' \\ Abdullah A Alzahrani ${ }^{2}$ \\ Wesal Y Alalayet ${ }^{2}$ \\ Abdulrahman A Alkharashi ${ }^{2}$ \\ Mohammed N Alarifi' \\ 'Department of Clinical Pharmacy, \\ College of Pharmacy, King Saud \\ University, Riyadh, Saudi Arabia; ${ }^{2}$ King \\ Saud University Medical City, Riyadh, \\ Saudi Arabia
}

Purpose: Warfarin is an affordable drug used for numerous indications, and still a favorable choice for patients with a history of bleeding from direct oral anticoagulants or presence of valvular heart diseases. However, warfarin requires regular international normalized ratio (INR) monitoring for safety and efficacy. Warfarin's efficacy and safety is correlated with actual time spent within the therapeutic INR. Time in therapeutic range (TTR) is an estimate that measures the percentage of actual time spent within the therapeutic INR. Our aim was to investigate differences in anticoagulation control of warfarin using TTR between pharmacists and other health-care providers.

Methods: This prospective observational study was conducted in an ambulatory-care setting of a tertiary hospital to compare anticoagulation management using TTR between clinics run by pharmacists versus other health-care providers.

Results: A total of 62 patients were enrolled: 33 in the pharmacist-led clinic and 29 in the physician-led clinic. TTR levels were statistically higher among patients in the pharmacistled clinic than than the physician-led clinic $(87.27 \% \pm 3.82 \%$ and $52.48 \% \pm 5.49 \%$, respectively; $p<0.001)$. For 27 patients followed retrospectively by physicians and prospectively by clinical pharmacists, TTR was statistically higher during clinical pharmacists' care $(91.70 \%$ $\pm 2.93 \%$ versus $61.39 \% \pm 5.11 \%$, respectively; $p<0.001$ ). During the study, approximately $82 \%$ of patients reached their target INR in the pharmacist-led clinic compared to $24 \%$ in the physician-led clinic.

Conclusion: The findings of our study found that patients followed in the pharmacist-led clinic had higher TTR levels than those followed in the physician-led clinic.

Keywords: warfarin, time in therapeutic range, pharmacists, physicians, clinic, TTR, INR, anticoagulation, Saudi Arabia

\section{Introduction}

Warfarin is a high-alert medication and a commonly prescribed medicationused by numerous patients for various indications. Due to the many drug-drug, drug-food, and drug-disease interactions and genetic influence on warfarin pharmacodynamics/pharmacokinetics, warfarin doses are highly variable among individuals and regular dosage adjustment inevitable. ${ }^{1}$ Despite the current availability of direct oral anticoagulants (DOACs), which have fewer drug-food interactions with unneeded regular bloodcoagulation monitoring, the risk of bleeding associated with some DOACs, burden of high cost, and presence of valvular heart diseases limit the clinical utilization of DOACs, making warfarin still a favorable choice for both clinicians and patients. ${ }^{2,4}$
Correspondence: Sultan Alghadeeer PO Box 2475, Riyadh II45, Saudi Arabia Tel +966-II-467-7497

Fax+966II-4674229

Email salghadeer@ksu.edu.sa
Risk Management and Healthcare Policy 2020:13 || 175-II79

Submit your manuscript DovePress if in $v$ 
Anticoagulation with warfarin requires regular international normalized ratio (INR) readings for safety and efficacy monitoring. ${ }^{1}$ The goal of therapy is specified by a therapeutic INR range that is determined based on the clinical indication. Having the INR reading within the therapeutic INR range for particular diseases during follow-up provides an objective parameter for the efficacy and safety of warfarin. However, individual INR readings do not reflect the actual time spent in the therapeutic INR range during the period between visits. Warfarin's efficacy and safety is correlated with actual time spent within the therapeutic INR. Time in therapeutic range (TTR) is an estimate that measures the percentage of actual time spent within the therapeutic INR. ${ }^{5}$ The optimal clinical benefit of warfarin therapy is achieved only when the patient's INR is kept within the therapeutic range, ideally during the entire period. Maintaining TTR at or above $70 \%$ is associated with clinical benefit in terms of efficacy and safety. ${ }^{6}$

The anticoagulation control of warfarin therapy is affected by the clinical judgment of the treating clinician. Pharmacists are known for their role in patient counseling, dose adjustment, and identifying drug-drug, drug-food, and drug-disease interactions. ${ }^{7}$ Our study aimed to investigate differences in anticoagulation control of warfarin utilizing a TTR approach in clinics managed by a clinical pharmacist compared with clinics managed by other health-care providers.

\section{Methods}

This prospective observational study ran from June 2017 to July 2018 at King Saud University Medical City (KSUMC) in Riyadh, capital of Saudi Arabia. All adult patients being followed in outpatient anticoagulation clinics to be coagulated with warfarin were candidates for inclusion. Patients on any DOACs, heparin products, or other anticoagulants, or had been admitted during follow-up, visited the emergency department for bleeding that resulted in warfarin being withheld, or crossed over between the study clinics during the study period were excluded. The study was approved by the institutional review board at KSUMC (E-17-2547). Patient consent to review their medical records was not required by the board, because this was an observational, noninterventional prospective study only to collect particular human data and subjects would receive the standard of care regardless. In addition, these patient-related data were collected confidentially and in compliance with the Declaration of Helsinki.
Patients were followed in two anticoagulation clinics: one run by clinical pharmacists and the other by physicians. Ambulatory-care clinical pharmacists supervise the pharmacist-led clinic and hematology consultants the physician-led clinic. In each clinic, patients were randomly allocated and followed for 12 months to assess qualitative differences in anticoagulation control in the two clinics. Patients were followed with INR measurements and warfarin-dose assessment at each visit. Other information were collected through patient-chart reviews, including demographics, disease status and renal function. An institutional protocol for the frequency of INR measurement in cases of dose adjustments was available and approved where the study was conducted. This protocol generally states that INR measurements after dose adjustment should be performed more frequently; however, the frequency of visits is determined and judged by treating clinicians and range from weekly to every 3 months according to the patient's clinical status. For each patient, at least three to four INR readings were collected to be included in calculations using Rosendaal linear interpolation and measured with a computer-based program. Rosendaal linear interpolationis a method used by the vast majority of clinical researchers to calculate TTR. ${ }^{8}$ Good anticoagulation control is defined as TTR $\geq 70 \%$, while poor anticoagulation control is defined as TTR $<70 \%$. The INR readings included in the TTR measurement were at least 1 month apart (this was to make sure that the INR was stable), and readings separated by more than 1 month were allowed.

Our primary outcome was differences in TTR, measured using Rosendaal linear interpolation, between the pharmacist-led clinic and the physician-led clinic. Patients who were seen by clinical pharmacists during our study were followed retrospectively to measure TTR during the period when the same patients were followed by physicians, and were included as a secondary outcome. Data was analyzed by SPSS version 25.0. Continuous variables are presented as means $\pm \mathrm{SD}$ and categorical variables as numbers and percentages. Continuous variables were compared between groups using Students' $t$-test or Mann-Whitney $U$ test. Categorical variables were compared using $\chi^{2}$ or Fisher's exact test. $p<0.05$ was considered significant.

\section{Results}

A total of 62 patients were enrolled from June 2017 to July 2018: 33 patients were followed by the pharmacist-led 
clinic and 29 by the physician-led clinic. Mean age of patients was $53 \pm 2.6$ years and $53.2 \%$ were male. Baseline characteristics are shown in Table 1. Apart from age and diabetes, which weresignificantly different between patients followed by clinical pharmacists versus those followed by physicians, all other demographic data were comparable between the two groups.

The main indication for warfarin use was mitral valve replacement $(60 \%$ in pharmacist-led arm and $31 \%$ in physician-led arm) followed by venous thromboembolism treatment (15\% in the pharmacist-led arm and around $17 \%$ in the physician-led arm). Other indications are shown in Table 2. Eighteen patients in the pharmacist-led clinic versus 16 in the physician-led clinic had their doses of warfarin changed over the study period $(55 \%$ in pharmacist-led clinic versus $55 \%$ in physician-led clinic, $p=0.961$ ). The number of changed doses of warfarin occurred in pharmacist-led clinics compared to physicianled clinics is listed in Table 3.

Mean TTR level was significantly higher among patients in the pharmacist-led clinic than the physician-led clinic $(87.27 \% \pm 3.82 \%$ and $52.48 \% \pm 5.49 \%$, respectively; $p<0.001$; Figure 1). Secondary analysis was conducted among the same patients $(\mathrm{n}=27)$ followed retrospectively by physicians and prospectively by clinical pharmacists,

Table I Baseline characteristics

\begin{tabular}{|l|l|l|l|l|}
\hline \multicolumn{2}{|l|}{} & $\begin{array}{l}\text { Pharmacist } \\
(\mathbf{n = 3 3 )}\end{array}$ & $\begin{array}{l}\text { Physician } \\
(\mathbf{n = 2 9 )}\end{array}$ & P-value \\
\hline Gender, n (\%) & Male & $14(42 \%)$ & $8(27 \%)$ & 0.223 \\
& Female & $19(58 \%)$ & $21(83 \%)$ & 0.223 \\
\hline Age, years (mean \pm SD) & $58.76 \pm 2.07$ & $46.86 \pm 3.6$ & 0.002 \\
Htn, n (\%) & $14(42 \%)$ & $9(31 \%)$ & 0.354 \\
DM, n (\%) & $14(42 \%)$ & $3(10 \%)$ & 0.009 \\
IHD, n (\%) & $6(18 \%)$ & $6(20 \%)$ & 0.803 \\
CrCl, mL/min (mean + SD) & $87.55 \pm 6.97$ & $103.54 \pm 8.89$ & 0.157 \\
FU, months (mean $\pm S D)$ & $7.88 \pm 0.41$ & $8.55 \pm 0.38$ & 0.238 \\
\hline
\end{tabular}

Abbreviations: $\mathrm{CrCl}$, creatinine clearance; DM, diabetes; $\mathrm{FU}$, follow-up; $\mathrm{Htn}$, hypertension; IHD, ischemic heart disease.

Table 2 Indications of warfarin

\begin{tabular}{|l|l|l|l|l|l|}
\hline & MVR* & AVR & $\begin{array}{l}\text { DVTI } \\
\text { PE }\end{array}$ & Afib & $\begin{array}{l}\text { SLEI } \\
\text { APS* }\end{array}$ \\
\hline $\begin{array}{l}\text { Pharmacists }(\mathrm{n}=33) \\
\text { Physicians }(\mathrm{n}=29)\end{array}$ & $\begin{array}{l}20(60 \%) \\
9(31 \%)\end{array}$ & $\begin{array}{l}3(9 \%) \\
4(13 \%)\end{array}$ & $\begin{array}{l}5(15 \%) \\
5(17 \%)\end{array}$ & $\begin{array}{l}4(12 \%) \\
4(13 \%)\end{array}$ & $\begin{array}{l}7(3 \%) \\
7(24 \%)\end{array}$ \\
\hline
\end{tabular}

Note: ${ }^{*} p=0.002$ ).

Abbreviations: Afib, atrial fibrillation; AVR, aortic valve replacement; DVT/PE, deep- vein thrombosis/pulmonary embolism; MVR, mitral valve replacement; SLE/ APS, systemic lupus erythematosus/antiphospholipid syndrome.
Table 3 Changes in warfarin dose

\begin{tabular}{|l|l|l|l|}
\hline $\begin{array}{l}\text { Number of changed } \\
\text { doses per patient }\end{array}$ & $\begin{array}{l}\text { Pharmacist- } \\
\text { led clinics }\end{array}$ & $\begin{array}{l}\text { Physician- } \\
\text { led clinics }\end{array}$ & Total \\
\hline No change & 15 & 13 & 28 \\
I & 15 & 6 & 21 \\
2 & 2 & 8 & 10 \\
3 & 1 & 1 & 2 \\
4 & 0 & 1 & 1 \\
\hline Total & 33 & 29 & 62 \\
\hline
\end{tabular}

Note: $p$-value for changed doses between pharmacists and physicians was 0.961 .

revealing that TTR was significantly higher duringclinical pharmacist care $(91.70 \% \pm 2.93 \%$ versus $61.39 \% \pm 5.11 \%$, respectively; $p<0.001$; Figure 2). On follow-up visits during the study period, approximately $82 \%$ of the patient reached their target INR in the pharmacist-led clinic compared to $24 \%$ in the physician-led clinic (Figure 3). No bleeding or thrombotic events were reported during the study period.

\section{Discussion}

Some national guidelines set a target of TTR at $60 \%$ or above, ${ }^{9,10}$ while others define a TTR range of $58 \%-65 \%$ as acceptable to maximize warfarin efficacy and decrease

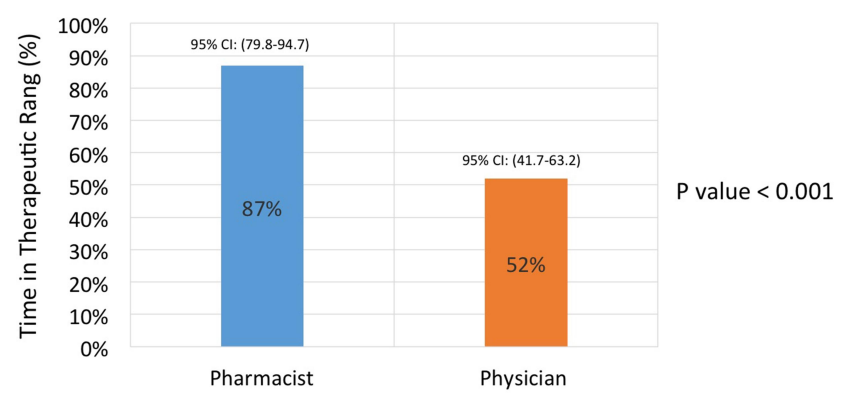

Figure I Difference in time in therapeutic range between pharmacist-led and physician-led clinics.

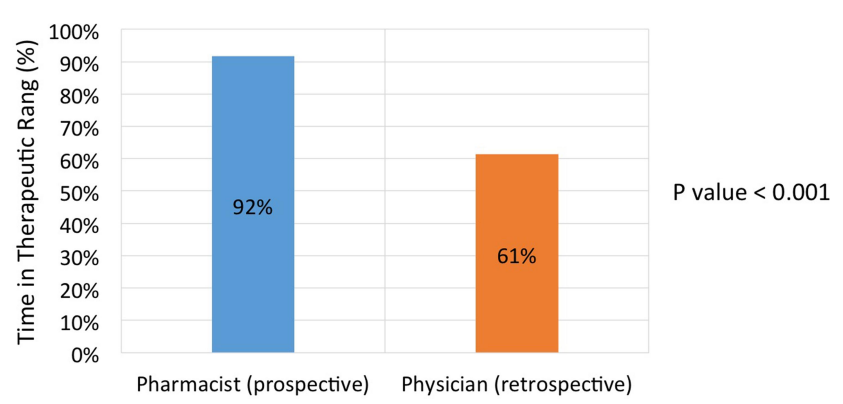

Figure 2 Difference in time in therapeutic range between pharmacist-led clinic (prospective) and physician-led clinic (retrospective). 


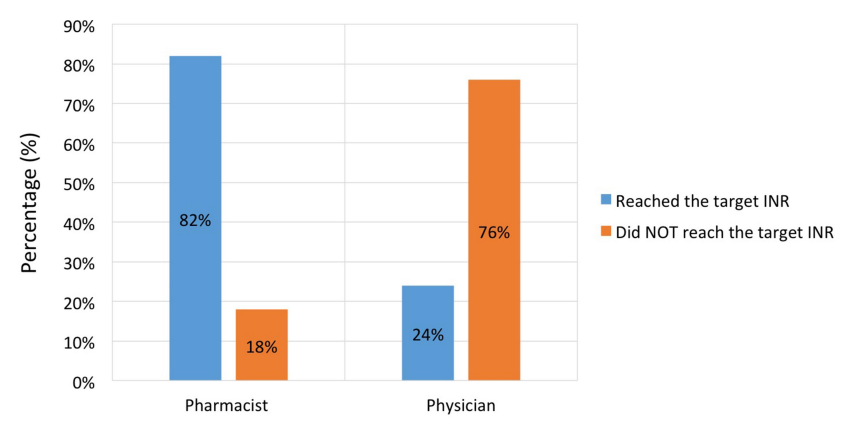

Figure 3 Percentage of patients reaching their target INR. Abbreviation: INR, international normalized ratio.

its toxicity. ${ }^{11}$ Other national guidelines and studies report that maintaining TTR $\geq 70 \%$ is associated with clinical benefit in terms of efficacy and safety. ${ }^{8,12}$ In our study, patients followed in the pharmacist-led clinic had higher TTR (87.27\%), beyond TTRs in different guidelines, than those followed in the physician-led clinic (52.48\%). Also, more patients $(82 \%)$ followed in the pharmacist-led clinic reached the targeted INR than patients in the physician-led clinic (24\%). These results are consistent with other studies conducted globally. A study conducted in an ambulatory-care setting in the US found that pharmacists achieved higher TTR than physicians (66\% vs $56.6 \%$, $p=0.028) .{ }^{13}$ Similar results were seen among Canadian patients, as the TTR for pharmacists was $73 \%$ compared to $65 \%$ for physicians $(p<0.0001) .{ }^{14}$ The superiority of pharmacists in anticoagulation management over physicians utilizing TTR has been reported in clinical trials. Trials conducted in Canada and Hong Kong found that pharmacists attained significantly higher TTR $(82 \%$ and 64\% among Canadian and Hong Kong patients, respectively) than physicians ( $76 \%$ and $59 \%$ among Canadian and Hong Kong patients, respectively). ${ }^{15,16}$ Furthermore, pharmacist-led anticoagulation clinics were able to improve poor control of warfarin therapy, with TTR $<50 \%$. A study conducted in Brazil assigned patients with TTR $<50 \%$ to anticoagulation clinics managed by pharmacists. These clinics had improved TTR readings significantly at 12 weeks postassignment compared to the previous year's TTR. ${ }^{17}$

Superior pharmacist management of anticoagulation clinics may result from the availability of pharmacists for more frequent coagulation tests, as this is assured by the number of INR readings compared to physicians. In a study conducted by Young et al and another by Shah et al, the number of INR-monitoring tests per year was higher among pharmacists than physicians. ${ }^{13,14}$ Also, pharmacist recommendations diminished the number of medication errors, drug interactions, and INR reading outrange, which in turn lead to higher TTR values and greater patients medication adherence. ${ }^{18} \mathrm{~A}$ study by Aidit et al indicated that pharmacists had a positive influence on management by implementing warfarin medication-adherence clinics ith pharmacists who dedicated some of their time to education, counseling, and dealing with adherence issues. ${ }^{19}$ Furthermore, pharmacists' accessibility for questions and communication influenced the excellent management of anticoagulation that facilitated the establishment of a community pharmacist-led anticoagulation-management service in New Zealand and the development of pharmacist community clinics for anticoagulation in some parts of the US and UK. ${ }^{10,20,21}$

In addition to the good management of pharmacist-led anticoagulation clinics, pharmacists in these clinics attain great patient satisfaction by applying valuable interpersonal communication skills and providing essential related information, gaining confidence in their capabilities in dealing with patients from other health-care providers, and providing the service in a cost-effective manner. ${ }^{10,21}$ A survey conducted among patients attending community anticoagulation clinics found that pharmacists scored highly on communication ( $99.4 \%-99.9 \%$ of patients) and information provided (63.1\%-94.5\% of patients) on such indications as adverse effects, dose adjustment, and medication use. ${ }^{21}$ The health-care cost of pharmacist-led anticoagulation clinics seems lower. It has been estimated that the health-care cost of a pharmacist-led clinic is US $\$ 908.16$ compared to $\$ 1,301.76$ per patient per year for a physician-led clinic among patients in New Zealand and $\$ 180.21$ compared to $\$ 352.29$ per patient per 6 months among patients in Malaysia. ${ }^{10,22}$

This study was conducted in single center on a small number of patients, because we followed patients managed solely by either pharmacists or physicians during the whole period of our study. Also, the duration of visits and the education or information provided were not reported. However, the prospective design, parallel comparison, and relatively long followup make our study different from most published retrospective studies in the literature. In addition, to our knowledge, this is the first study to evaluate the impact of clinical pharmacists compared to physicians in anticoagulation clinics in an ambulatory-care setting in Saudi Arabia. 


\section{Conclusion}

Our study found that patients followed in the pharmacistled clinic had higher TTR levels than those followed in the physician-led clinic.

\section{Acknowledgment}

The authors would like to thank the Deanship of Scientific Research and Research Center at the College of Pharmacy in King Saud University, Saudi Arabia.

\section{Disclosure}

The authors report no conflicts of interest in this work.

\section{References}

1. Coumadin (warfarin Sodium). Prescribing Information. Princeton (NJ): Bristol-Myers Squibb, Inc.; 2017.

2. Holster IL, Valkhoff VE, Kuipers EJ, Tjwa ET. New oral anticoagulants increase risk for gastrointestinal bleeding: a systematic review and meta-analysis. Gastroenterology. 2013;145:105-12.e15. doi:10.1053/j. gastro.2013.02.041

3. Zheng Y, Sorensen SV, Gonschior A-K, et al. Comparison of the cost-effectiveness of new oral anticoagulants for the prevention of stroke and systemic embolism in atrial fibrillation in a UK setting. Clin Ther. 2014;36(12):2015-28.e2. doi:10.1016/j.clinthera.2014.09.015

4. Vinogradova Y, Coupland C, Hill T, Hippisley-Cox J. Risks and benefits of direct oral anticoagulants versus warfarin in a real world setting: cohort study in primary care. BMJ. 2018;362:k2505. doi:10.1136/bmj.k2505

5. Rosendaal FR, Cannegieter SC, van der Meer FJ, Briët E. A method to determine the optimal intensity of oral anticoagulant therapy. Thromb Haemost. 1993;69(3):236-239. doi:10.1055/s-0038-1651587

6. Morgan CL, McEwan P, Tukiendorf A, et al. Warfarin treatment in patients with atrial fibrillation: observing outcomes associated with varying levels of INR control. Thromb Res. 2009;124(1):37-41. doi:10.1016/j.thromres.2008.09.016

7. Schnipper JL, Kirwin JL, Cotugno MC, et al. Role of pharmacist counseling in preventing adverse drug events after hospitalization. Arch Intern Med. 2006;166(5):565-571. doi:10.1001/archinte.166.5.565

8. Canadian Agency for Drugs and Technologies in Health (CADTH). Optimal Warfarin Management for the Prevention of ThromboembolicEvents in Patients with Atrial Fibrillation: A Systematic Review of theClinical Evidence [Internet]. Ottawa (ON): Canada; November, 2011. CADTH 290 Optimal Use Report, No. 1.2A. Available from: https://www.ncbi.nlm.nih.gov/books/ NBK169490/. Accessed August 5, 2020.
9. Thrombosis Canada. Warfarin: Management of Out-Of-Range INRs. Whitby, ON: Thrombosis Canada; 2015. Available from: http://throm bosiscanada.ca/clinicalguides/\#. Accessed January 27, 2010.

10. Shaw J, Harrison J, Harrison JE. Community Pharmacist-Led Anticoagulation Management Service: Final Report. Wellington, New Zealand: Health Workforce New Zealand; Septmeber; 2011.

11. Ageno W, Gallus AS, Wittkowsky A, et al. Oral anticoagulant therapy: antithrombotic therapy and prevention of thrombosis, 9th ed: American College of chest physicians evidence-based clinical practice guidelines. Chest. 2012;141(2):e44s-e88s. doi:10.1378/chest.11-2292

12. British committee for standards in haematology: guidelines for point of care testing. 2019.

13. Shah KJ, Mansukhani R, Bloomstein D, Serra M. Outcomes of a pharmacist managed anticoagulation service. IOSR $J$ Pharm. 2016;6(10):62-67.

14. Young S, Bishop L, Twells L, Dillon C, Hawboldt J, O'Shea P. Comparison of pharmacist managed anticoagulation with usual medical care in a family medicine clinic. BMC Fam Pract. 2011;12(1):88. doi:10.1186/1471-2296-12-88

15. Wilson SJ, Wells PS, Kovacs MJ, et al. Comparing the quality of oral anticoagulant management by anticoagulation clinics and by family physicians: a randomized controlled trial. CMAJ. 2003;169:293-298.

16. Chan FW, Wong RS, Lau WH, Chan TY, Cheng G, You JH. Management of Chinese patients on warfarin therapy in two models of anticoagulation service - a prospective randomized trial. $\mathrm{Br} J \mathrm{Clin}$ Pharmacol. 2006;62:601-609. doi:10.1111/j.1365-2125.2006.02693.x

17. Marcatto LR, Sacilotto L, Tavares LC, et al. Pharmaceutical care increases time in therapeutic range of patients with poor quality of anticoagulation with warfarin. Front Pharmacol. 2018;9:1052. doi:10.3389/fphar.2018.01052

18. Lee T, Davis E, Kielly J. Clinical impact of a pharmacist-led inpatient anticoagulation service: a review of the literature. Integr Pharm Res Pract. 2016;5:53-63. doi:10.2147/IPRP.S93312

19. Aidit S, Chang Soh Y, Seng Yap C, et al. Effect of standardized warfarin treatment protocol on anticoagulant effect: comparison of a warfarin medication therapy adherence clinic with usual medical care. Front Pharmacol. 2017;8:637. doi:10.3389/fphar.2017.00637

20. Coleman B, Martin C, Barber N, Patterson D. An evaluation of the safety and acceptability of an anticoagulation clinic in a community pharmacy setting - a pilot study. Pharm J. 2004;273:822-824.

21. Ingram SJ, Kirkdale CL, Williams S, et al. Moving anticoagulation initiation and monitoring services into the community: evaluation of the brighton and hove community pharmacy service. BMC Health Serv Res. 2018;18(1):91. doi:10.1186/s12913-018-2901-8

22. Thanimalai S, Shafie AA, Hassali MA, Sinnadurai J. Costeffectiveness of warfarin medication therapy adherence clinic versus usual medical clinic at Kuala Lumpur Hospital. Value Health Reg Issues. 2018;15:34-41.
Risk Management and Healthcare Policy is an international, peerreviewed, open access journal focusing on all aspects of public health, policy, and preventative measures to promote good health and improve morbidity and mortality in the population. The journa welcomes submitted papers covering original research, basic science, clinical \& epidemiological studies, reviews and evaluations, guidelines, expert opinion and commentary, case reports and extended reports. The manuscript management system is completely online and includes a very quick and fair peer-review system, which is all easy to use. Visit http://www.dovepress.com/testimonials.php to read real quotes from published authors. 